See discussions, stats, and author profiles for this publication at: https://www.researchgate.net/publication/273575464

\title{
Estimating a Mean-Path from a Set of 2-D Curves
}

Conference Paper in Proceedings - IEEE International Conference on Robotics and Automation · August 2015

DOI: 10.1109/ICRA.2015.7139467

CITATIONS

4

4 authors:

Amir Ghalamzan

University of Lincoln

23 PUBLICATIONS 99 CITATIONS

SEE PROFILE

Marcello Restelli

Politecnico di Milano

100 PUBLICATIONS 747 CITATIONS

SEE PROFILE
READS

63

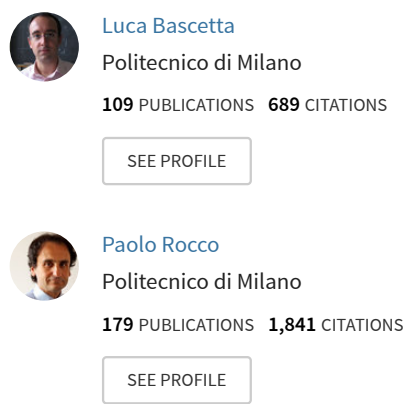

Some of the authors of this publication are also working on these related projects:

Project VR Driving Simulator for analysis of driver behavior View project

Project Motion Planning for mobile robots under kynodynamic, actuation and safety constraints View project 


\title{
Estimating a Mean-Path from a Set of 2-D Curves
}

\author{
Amir M. Ghalamzan E., Student member, IEEE, Luca Bascetta, member, IEEE, \\ Marcello Restelli, member, IEEE, and Paolo Rocco, member, IEEE
}

\begin{abstract}
To perform many common industrial robotic tasks, e.g. deburring a work-piece, in small and medium size companies where a model of the work-piece may not be available, building a geometrical model of how to perform the task from a data set of human demonstrations is highly demanded. In many cases, however, the human demonstrations may be sub-optimal and noisy solutions to the problem of performing a task. For example, an expert may not completely remove the burrs that result in deburring residuals on the work-piece. Hence, we present an iterative algorithm to estimate a noise-free geometrical model of a work-piece from a given dataset of profiles with deburring residuals. In a case study, we compare the profiles obtained with the proposed method, nonlinear principal component analysis and Gaussian mixture model/Gaussian mixture regression. The comparison illustrates the effectiveness of the proposed method, in terms of accuracy, to compute a noise-free profile model of a task.
\end{abstract}

\section{INTRODUCTION}

In-contact tasks, such as deburring [1], are among the most common robotic tasks. In case of frequent changes in a production line in small and medium size companies, where a model of work-piece may not be available, Robot Programming by Demonstration (RPD) is potentially useful to obtain a nominal profile model of the work-piece from a set of expert demonstrations. RPD simplifies the complex process of programming a robot and reduces the programming cost. We assume that an expert performance is suboptimal and she/he cannot perfectly remove the burrs of a work-piece resulting in deburring residuals. Hence, we propose an approach that estimates a nominal profile of the work-piece from a set of sub-optimal expert demonstrations (Figure 1).

The problem of a robot learning how to perform the task of deburring from human demonstrations has been previously studied [2], [3]. Automatic deburring by using robots has attracted much research interest due to the increase of production speed and operator's hazard reduction. For example, the relation between the tool feed rate and burr characteristics was stored in an associative memory represented by a neural network in [2]. The obtained neural network was then used to produce the feed rate for a new burr characteristic, where the burr size was measured using a laser sensor and assuming a

The research leading to these results has received funding from the European Community's Seventh Framework Programme FP7/2007-2013 - Challenge 2 - Cognitive Systems, Interaction, Robotics - under grant agreement No 231143 - ECHORD (Experiment FIDELIO).

Amir M. Ghalamzan E., Luca Bascetta, Marcello Restelli, and Paolo Rocco are with the Politecnico di Milano, Dipartimento di Elettronica, Informazione e Bioingegneria, Piazza Leonardo da Vinci 32, 20133 Milan, Italy. (E-mail: \{amirmasoud.ghalamzanesfahani, luca.bascetta, marcello.restelli, and paolo.rocco\}@polimi.it) model of the work-piece is known. Ziliani et al. [4] used a hybrid force/velocity control method along with a new design of the deburring tool with two ball bearings to avoid penetration of the tool in the work-piece. Aertbelin et al. [5] modeled the expert actions as an impedance controller with adaptive parameters in accordance with burr characteristics. A neural network was then employed to learn the nonlinear relation between the parameters and the burr characteristics.

The problem of learning the required force and feed rate has been studied in prior works, from human demonstrations [2], [5] to adaptation during execution through intelligent active control [3], [6] or Reinforcement Learning [7], [8] However, prior works did not take into account the problem of obtaining a profile model from a set of profiles collected after the deburring operation was executed. In fact, they assumed that a nominal profile of a work-piece either is available or is not needed due to the use of mechanical constraints [4]. In case that the profile model of the workpiece is not available a priori, one may benefit from existing RPD methods to obtain a nominal profile model from expert demonstrations.

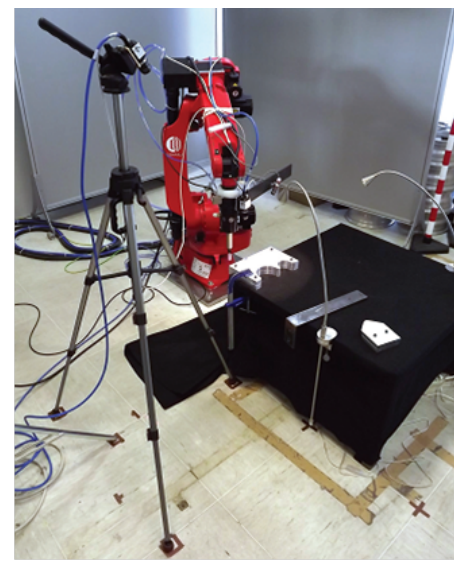

Fig. 1. A robotic deburring setup.

\section{A. Related works}

In the context of RPD, Gaussian Mixture Model/Gaussian Mixture Regression (GMM/GMR), a nonlinear regression method, has been studied to compute a generalized trajectory/path from a set of demonstrations [9]. Moreover, in order to reduce the dimensionality of the demonstrated dataset and to remove the noise from it, Calinon et al. [10] used Principal Component Analysis (PCA) before computing the generalized trajectory by GMM/GMR. The optimal number 
of Gaussian Mixture Model (GMM) components can be estimated by different criteria, such as Bayesian Information Criterion (BIC) [9]. Cho [11] proposed an online version of GMM/GMR, that automatically determines the number of Gaussian components during learning when a new teaching trial is available.

In another line of research, in order to capture and remove nonlinear correlation between data points lying on a nonlinear manifold, Nonlinear Principal Component Analysis (NLPCA) has been proposed [12], [13], in analogy with standard PCA.

In a dataset related to a deburring example, we assume that every single demonstrated work-piece profile is strongly correlated with the nominal one. Hence, a nonlinear principal component of the demonstrated dataset is an estimation of the nominal profile of the work-piece, and captures the most variation of the dataset.

In this work, we present a metric that allows us to compare the precision of the profiles computed by NLPCA [12], GMM/GMR [10]. We show that the precision of these methods depends on the shape of the profile. Consequently, we propose an iterative algorithm computing a nonlinear profile that minimizing the proposed metric. This algorithm results in a smaller error, with respect to GMM/GMR and NLPCA, for different shapes of the profile. In fact, though increasing the number of components in the GMM/GMR model improves the accuracy of the estimated profile, the comparison illustrates that the proposed approach outperforms the GMM/GMR and NLPCA in terms of accuracy.

The remainder of this paper is organized as follows. In Section II, the problem definition and formulation are introduced. Section III presents a transformation from the Cartesian space to a new space that, as described in Section IV, ease the computation of the Mean-Path. In Section V, a case study, based on a deburring example with real burr profiles, is used to compare GMM/GMR and NLPCA with the proposed approach.

\section{PROBLEM DEFINITION}

Consider a set of $n$ continuous 2-D curves $\beta_{j}$ (Fig. 2)

$$
X=\left\{\beta_{1}(s), \beta_{2}(s), \ldots, \beta_{n}(s)\right\}
$$

where $s$ is the arc length.

Although curves as expressed in eq. (1) are continuous, they are, usually, available only through a set of collected sample points. Hereinafter, we call a sequence of sample points, collected from a continuous curve $\beta$, a path $\zeta$, and we assume that all the paths $\left(\zeta_{j} \in \mathscr{X}, j=1,2, \ldots, n\right)$ are represented by the same number of sample points

$$
\zeta_{j}=\left\{P_{j, i} \in \mathbb{R}^{2}, P_{j, i}=\beta_{j}\left(s_{i}\right), \forall i=1, \ldots, m\right\}
$$

In the following, we always considered a path as a sequence of points unless differently stated.

In order to formalize the definition of Mean-Path, an Euclidean distance (Fig. 2) between a point on a path, called Reference-Path, and another path is introduced in the following.

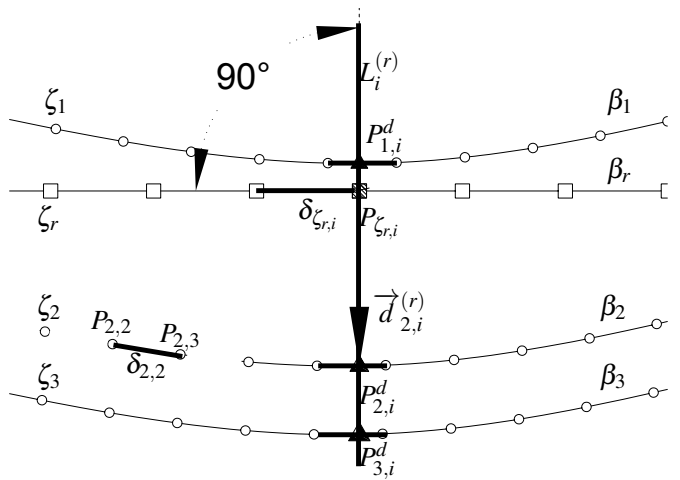

Fig. 2. A set of four 2-D curves (continuous paths $\beta$ and their collected data points $\zeta$, shown by circle signs); Reference-Path $\beta_{r}$ and its data points $\zeta_{r}$ shown with square signs; line segment $\delta_{2,2}=\overline{P_{2,2}, P_{2,3}}$ linearly approximates the continuous path $\beta_{2}$ between $P_{2,2}$ and $P_{2,3}$; line $L_{i}^{(r)}$ is perpendicular to $\delta_{\zeta_{r, i}}$; intersections of $L_{i}^{(r)}$ and each $\Delta_{\zeta_{j}}$, denoted by $P_{j, i}^{d}, \forall j=1,2,3$ (marked with triangle sings). Vector $d_{2, i}^{(r)}$ determines the position of $P_{2, \zeta_{r, i}}^{d}$ relative to $P_{\zeta_{r, i}}$

\section{A. DISTANCE BETWEEN A REFERENCE-POINT AND A PATH}

Let $\zeta_{r}=\left\{P_{\zeta_{r}, 1}, \ldots, P_{\zeta_{r}, m}\right\}$ be a 2-D path, called ReferencePath, taken as a reference for distance computation. Further, $P_{\zeta_{r}, i}$ s are called Reference-Points (RPos).

A set of line segments, denoted by $\Delta_{\zeta_{j}}$, connecting two consecutive points of a path, is defined as follows

$$
\Delta_{\zeta_{j}}=\left\{\bar{\delta}_{j, 1}, \bar{\delta}_{j, 2}, \ldots, \bar{\delta}_{j, m-1}\right\} \quad \bar{\delta}_{j, i}=\overline{P_{j, i-1} P_{j, i}}
$$

where $\bar{\delta}_{j, i}$ is a line segment with the initial point $P_{j, i-1}$ and terminal point $P_{j, i}$. As a continuous path $\beta_{j}$ is usually not available, a piece-wise linear approximation of the sequence of points $\Delta_{\zeta_{j}}$ is used instead. For example, the subspace orthogonal to $\beta_{r}$ is approximated by the subspace orthogonal to each line segment of the Reference-Path, $\bar{\delta}_{\zeta_{r}, i}$ at a point $P_{\zeta_{r}, i}$, which is denoted by $\overleftrightarrow{L_{i}^{(r)}} \in \mathbb{R}^{n_{p}-1}$. In the 2-D case this subspace is a line called Perpendicular-Line, $\overleftrightarrow{L_{i}^{(r)}} \perp \bar{\delta}_{\zeta_{r}, i}$ (Fig. 2), and is characterized by the fact that the inner product of the unit vectors on $\overleftrightarrow{L_{i}^{(r)}}$ and $\bar{\delta}_{\zeta_{r, i}}$ is zero.

A similarity measure between a Reference-Path and another path in the set, denoted by $\vec{d}_{j, i}^{(r)}$, at each ReferencePoint $P_{\zeta_{r}, i} \forall i=1, \ldots, m$, is a vector whose absolute value is a distance between $P_{\zeta_{r}, i}$ and $\Delta_{\zeta_{j}}, \forall j=1, \ldots, n$, as follows

$$
\vec{d}_{j, i}^{(r)}:=\overrightarrow{P_{\zeta_{r}, i} P_{j, i}^{d}} \quad\left|\vec{d}_{j, i}^{(r)}\right|=\left\|P_{\zeta_{r}, i}-P_{j, i}^{d}\right\|
$$

where $P_{j, i}^{d}$ is an intersection between $\Delta_{\zeta_{j}}$ and $\overleftrightarrow{L_{i}^{(r)}}$ (Fig. 2). Intuitively, a local observer, moving along the ReferencePath, measures a distance from its location to $\Delta_{\zeta_{j}}$ at each $P_{\zeta_{r}, i}$ along $\overleftrightarrow{L_{i}^{(r)}}$.

In the case of more than one intersection between $\overleftrightarrow{L_{i}^{(r)}}$ and $\Delta_{\zeta_{j}}, \vec{d}_{j, i}^{(r)}$ with the minimum distance is chosen. Further, it is assumed that for a set of paths $\mathscr{X}$ and a considered $\zeta_{r}$ 
there is always an intersection between $\overleftrightarrow{L_{i}^{(r)}}$ and each $\Delta_{\zeta_{j}}$, otherwise the distance between $\Delta_{\zeta_{r}}$ and $\Delta_{\zeta_{j}}$ along $\overleftrightarrow{L_{i}^{(r)}}$ is assumed to be infinite ${ }^{1}$.

It is worth mentioning that the proposed definition of distance between $\Delta_{\zeta_{r}}$ and $\Delta_{\zeta_{j}}$ is not symmetric. This definition first determines a corresponding point on $\Delta_{\zeta_{j}} \mathrm{~s}$, namely $P_{j, i}^{d}$, with a point of $\zeta_{r}$, namely $P_{\zeta_{r}, i}$. Then the distance between these two points is taken as a similarity measure, computed by eq. (3).

For example, taking $\Delta_{\zeta_{r}}$ as Reference-Path $P_{j, i}^{d} \forall j=1, \ldots, n$ correspond to $P_{\zeta_{r}, i}$. However, for $\Delta_{\zeta_{j}}$ as Reference-Path, $P_{\zeta_{r}, i}$ does not necessarily correspond with $P_{j, i}^{d}$, as the sub-spaces orthogonal to $\Delta_{\zeta_{r}}$ and $\Delta_{\zeta_{j}}$ are not necessarily the same.

Note that, this similarity measure can be extended to higher dimensions by considering an intersection of a path and a subspace orthogonal to a Reference-Path.

\section{THE DISTANCE SPACE}

In this section, the similarity measure (eq. (3)) just introduced is used to transform paths based on their distances from the Reference-Path, in such a way that the information concerning the relative position of the considered path with respect to the Reference-Path is kept invariant.

Consider a Reference-Path $\zeta_{r} \subset \mathscr{X}$, a new representation of the set of paths $\mathscr{X}$, denoted by $\mathscr{Z}=\left\{\gamma_{1}, \ldots, \gamma_{n}\right\}$ where $\gamma_{j}=\left\{d_{j, \zeta_{r, 1}}, \ldots, d_{j, \zeta_{r, m}}\right\}$, is obtained by computing the similarity measure proposed in eq. (3) (Fig. 3). The vectors computed by eq. (3) constitute a bijective mapping, namely $\Phi^{2}$, from points of the data set in the Original-Space $\mathscr{X}$ to a set of points in the Distance-Space $\mathscr{Z}$, as follows

$$
\Phi\left(\mathscr{X}, \zeta_{r}\right): \mathscr{X} \stackrel{\overleftrightarrow{L_{i}^{(r)}} \perp \zeta_{r} \text { at } P_{\zeta_{r, i}}}{\longrightarrow} \mathscr{Z}
$$

Consequently, an inverse mapping, denoted by $\Psi$, from the Distance-Space, shown in Fig. 3(b), to the Original-Space, shown in Fig. 3(a), is also defined using the same $\zeta_{r}$.

It is worth mentioning that maps $\Phi$ and $\Psi$, based on Reference-Path $\zeta_{r}$, are symmetric, resulting in a one-by-one correspondence of each pair of points in $\mathscr{X}$ and $\mathscr{Z}$ (Fig. 3), as follows

$$
\mathscr{Z}=\Phi\left(\mathscr{X}, \zeta_{r}\right) \quad \mathscr{X}=\Psi\left(\mathscr{Z}, \zeta_{r}\right)
$$

Note also that the mapping from Original-Space to DistanceSpace $\Phi$ transforms $\zeta_{r}$ to the horizontal axis (Fig. 3(b)).

\section{MEAN-PATH}

To find a nonlinear path $\hat{\bar{\zeta}}$ along which the maximum variation of the dataset $\mathscr{X}$ is captured, we minimize the

\footnotetext{
${ }^{1}$ To be more precise, if no intersection is found, the intersections of $\overleftrightarrow{L_{i}^{(r)}}$ and either $\delta_{j, s t a r t}=\overrightarrow{P_{i, 2}, P_{i, t m p}}$, where $P_{i, t m p}=t \times \overrightarrow{P_{i, 2}, P_{i, 1}}$, or $\delta_{j, \text { end }}=$ $\overline{P_{i, \text { end }-1}, P_{i, t m p}}$, where $P_{i, t m p}=t \times \overline{P_{i, \text { end }-1}, P_{i, \text { end }}}$, are considered. If no intersection is found, not even with $\delta_{j, \text { end }}$ and $\delta_{j, \text { start }}$, an infinite distance is assigned to that point. The parameter $t$ has been chosen equal to 20 for the data sets in this paper.

${ }^{2}$ Notice that the mapping is invertible since it is bijective.
}

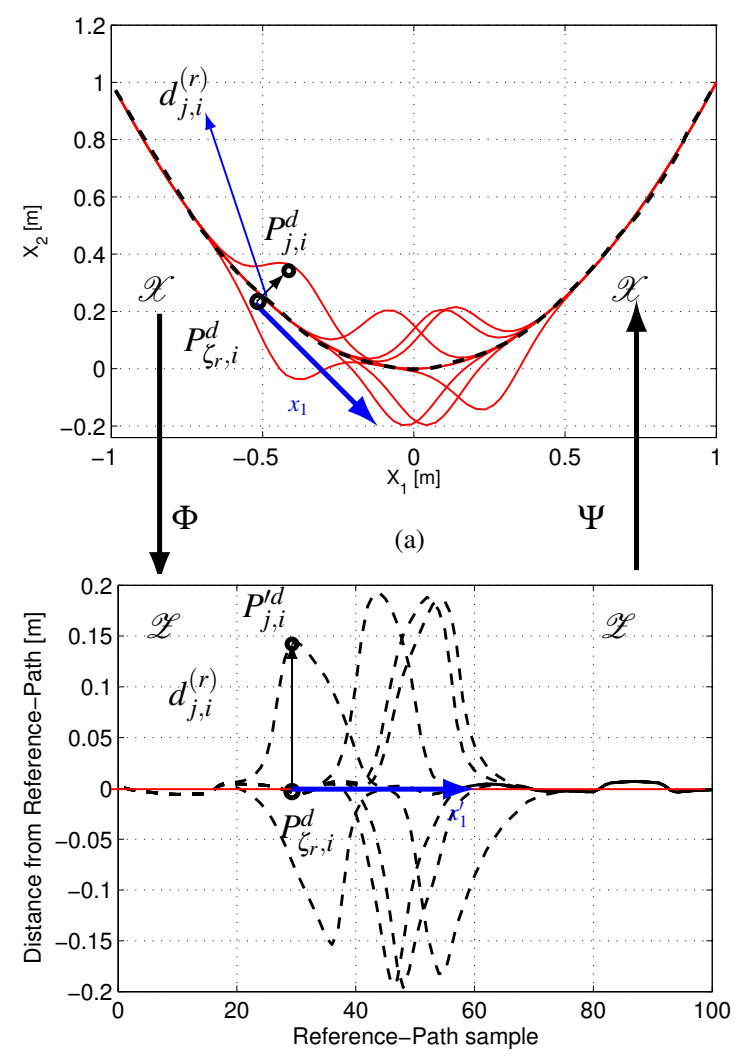

(b)

Fig. 3. A point $P_{j, i}^{d}$ in the Original-Space is transformed into a point in the Distance-Space and back, based on the considered Reference-Path (black dashed line). The Reference-Points, $P_{\zeta_{r}, i}^{d} \mathrm{~s}$ and $P_{j, i}^{d} \mathrm{~s}$ are marked with bold dots in both spaces. (a) A set of 2-D paths (red lines), a chosen Reference-Path $\zeta_{r}$ (black dashed line), the computed similarity metric $d_{j, i}^{(r)}$, and the local coordinate frame $x_{1}$ fixed at a Reference-Point and tangent to $\delta_{\zeta_{r}, i}$ (blue arrow). (b) The corresponding paths in Distance-Space and the corresponding local coordinate frame $x_{1}$ at the corresponding ReferencePoint (blue arrow). For better view, continuous representation of paths are shown.

trace of the data covariance in Distance-Space as follows

$$
\begin{aligned}
\hat{\bar{\zeta}}=\min _{\zeta_{r} \in \mathbb{R}^{m \times 2}} & \operatorname{tr}(\Sigma) \\
\text { s.t. } \quad \Sigma & =\mathbb{E}\left[\mathscr{Z}^{T} \mathscr{Z}\right] \\
\mathscr{Z} & =\Phi\left(\mathscr{X}, \zeta_{r}\right)
\end{aligned}
$$

The Reference-Path that minimizes the objective function in eq. (5) is called Mean-Path and is denoted by $\hat{\bar{\zeta}}$. In fact, a Distance-Space representation of the dataset based on the computed Mean-Path corresponds to align the first principal component of the dataset with the first main axis in the linear principal component analysis.

\section{A. Iterative algorithm to estimate the Mean-Path}

In this section, we present an iterative algorithm that computes a solution to eq. (5) for a set of paths, $\zeta_{j} \forall j=$ $1, \ldots, n$.

First, a path $\zeta_{r}^{(t)} \subset \mathscr{X}, t=1^{3}$, is picked as a reference

\footnotetext{
${ }^{3}$ The superscript $(t)$ stands for the iteration number, whereas the superscript with other letters such as $(r)$ stands for the values computed based on the considered Reference-Path.
} 
for Mean-Path computation. It is assumed that the paths are highly correlated with a nonlinear nominal path $\bar{\zeta}$, and each path has a small deviations from the nominal path at different points. Hence, $\zeta_{r}^{(1)} \subset \mathscr{X}$ is taken as an initial guess for the Mean-Path algorithm.

Then, the data set $\mathscr{X}$ is transformed into Distance-Space, as shown in Fig. 3, using eq. (4). Consequently, the residual of the dataset based on the considered Reference-Path is computed by eq. (5).

For each subspace $\overleftrightarrow{L_{i}^{(r)}}$ in Distance-Space, the points, $P_{j, i}^{d}, \forall j=1, \ldots, n$, are assumed to be generated randomly, therefore the expected value of $P_{j, i}^{d}, \forall j=1, \ldots, n$, is estimated by maximizing the likelihood of the sample points.

As for a set of normally distributed data points, the expected value minimizes the variance of the data points, a new point $P_{c, i}^{(t)}$ is computed minimizing the variance of all the corresponding intersection points $P_{j, i}^{d}, \forall j=1, \ldots, n$.

An innovation for each point of the Reference-Path is defined as $\vec{I}_{i}^{(t)}=P_{\zeta_{r}, i}-P_{c, i}^{(t)}$. Updating the origin of the coordinate systems by $\vec{I}_{i}^{(t)} \mathrm{s}$ can be considered as a gradient descent direction of the objective function in eq. (5). The sequence of $P_{c, i}^{(t)} \mathrm{s}$ is taken as a new Reference-Path $\zeta_{r}^{(t+1)}$, and the residual of the data based on $\zeta_{r}^{(t+1)}$ and $\zeta_{r}^{(t)}$ are compared to check the convergence condition, i.e. $\operatorname{tr}\left(\Sigma^{(t+1)}\right)<\operatorname{tr}\left(\Sigma^{(t)}\right)$. If the convergence condition is not satisfied a regularized innovation, $\lambda \vec{I}_{i}^{(t)}$, is used to compute the updated reference points, where $\lambda \in[0,1]$. This procedure is repeated with the new Reference-Path until converge to a solution.

A description of the iterative algorithm is reported in Algorithm 1 .

\section{CASE STUDY}

To evaluate the precision of the estimated profile by different methods, we present an example in which the nominal profile is known. We compare the computed path $\zeta_{r, i}^{(t)}$ to the nominal path $\bar{\zeta}$ using the following metric

$$
\varepsilon^{(t)}=\sum_{i=1}^{m_{1}}\left\|P_{\bar{\zeta}_{i}, \zeta_{r}^{(t)}}^{d}-P_{\bar{\zeta}, i}\right\| \quad P_{\bar{\zeta}_{i}, \zeta_{r}^{(t)}}=\overleftrightarrow{L}_{\bar{\zeta}_{i}} \cap \Delta_{\zeta_{r}^{(t)}}
$$

where $\zeta_{r}, \bar{\zeta} \in \mathbb{R}^{m_{1} \times 2}, \overleftrightarrow{L}_{\bar{\zeta}_{i}} \perp \Delta_{\bar{\zeta}}, P_{\bar{\zeta}, i}$ is the $i_{t h}$ sample point of $\bar{\zeta}, m_{1}$ is the number of profile sample points, $\Delta_{\bar{\zeta}}$ and $\Delta_{\zeta_{r}(t)}$ are computed by using eq. (2). This error is used to evaluate the accuracy of the computed Mean-Path.

Here we discuss the problem of automatically computing the deburring profile of a work-piece from a set of demonstrated profiles.

In order to have a robot that learns from demonstrations how to autonomously perform the deburring task, the robot must compute a nominal profile of the work-piece from a set of profiles collected after the deburring process. The nominal profile provides a baseline for another controller that computes the feed rate and velocity of the deburring tool. Therefore, an inaccurate estimation of the nominal profile results in a poor quality of the final work-piece, while

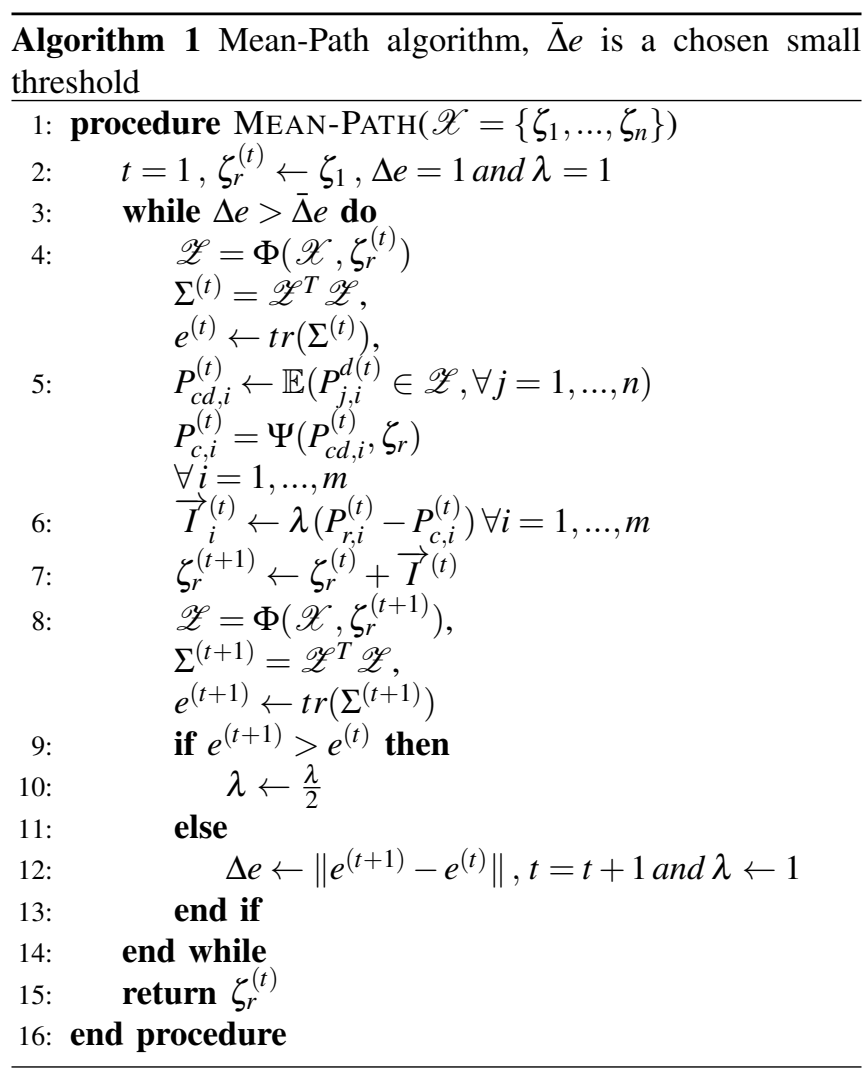

an accurate nominal profile results in minimum deburring residuals and minimum tool penetration.

We assume that an expert cannot always perfectly remove the burrs of a set of work-pieces, because human demonstration can be sub-optimal. Hence, a set of demonstrated profiles having deburring residuals with small sizes at different positions along the profile of the work-pieces is considered.

A dataset of nominal linear profiles and a dataset of nominal circular profiles with deburring residuals have been thus generated (see Fig. 5). To do so, a set of seven linear profiles with the same length and with different deburring residuals was first prepared, see Fig. 4(a). A camera and a laser projector were exploited through a visual triangulation algorithm to reconstruct the 2-D profiles of these deburring residuals (Fig. 4(b)). We consider that the nominal profile, linear or circular, consists of a number of profile segments with identical length. For each segment, a random binary generated number determines whether the deburring residual is added. Then a deburring residual profile is selected randomly and added to the nominal profile.

In order to estimate a nominal profile, we use the proposed algorithm, GMM/GMR [10] with different numbers of components, and NLPCA [12] with different modeling types.

The errors, $\varepsilon[\mathrm{mm}]$, between the computed and the nominal profile are reported in Table $\mathrm{I}^{4}$. For the linear profile, NLPCA and GMM/GMR result in error values of 127.1 and

\footnotetext{
${ }^{4}$ NA means that the corresponding error value is not available.
} 

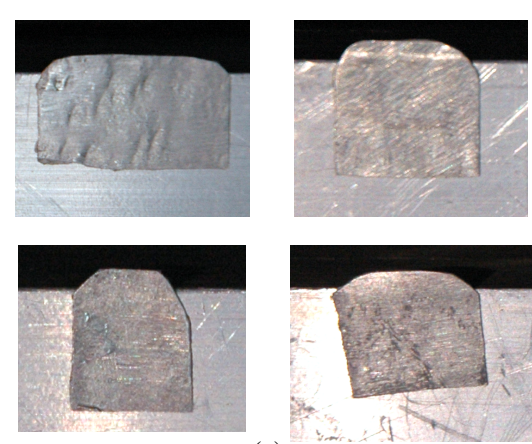

(a)

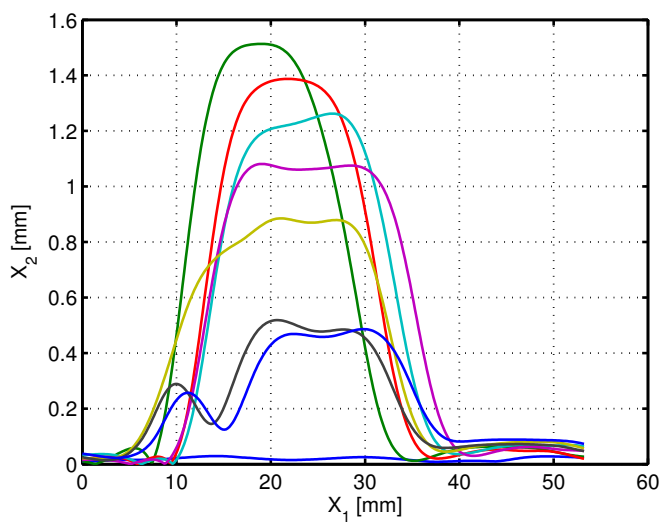

(b)

Fig. 4. (a) Sample work-pieces with the artificial deburring residuals. (b) The seven deburring residual models used to generate the data set.

$127.8[\mathrm{~mm}]$, while the Mean-Path algorithm error is $99.6[\mathrm{~mm}]$ (Fig. 6). The circular profile obtained with NLPCA and GMM/GMR results in a relative error of $1433.4[\mathrm{~mm}]$ and $75.9[\mathrm{~mm}]$ with respect to the circular nominal profile, but the profile computed with the Mean-Path algorithm results in an error of $75.3[\mathrm{~mm}]$ (Fig. 7). Although the circular profile obtained with GMM/GMR has almost similar error value compared to the one obtained with the Mean-Path algorithm, the work-piece will be damaged if the robot follows this profile during the execution of the deburring task, as this profile penetrates in the work-piece in some parts (see Figs. 6(b) and 7(b) in which the green shaded areas represent the nominal work-piece).

The aforementioned comparison illustrates the superiority of the proposed approach for both linear and circular profiles.

TABLE I

$\varepsilon[\mathrm{mm}]$ OF DEBURRING DATASET BY USING NLPCA, GMM/GMR AND MEAN-PATH FOR LINEAR/CIRCULAR PROFILE

\begin{tabular}{l|r|r}
\hline & symmetric & hierarchic \\
\hline circular/bottleneck & NA / NA & NA / NA \\
\hline circular/inverse & NA / 1433.4 & NA / 18534.5 \\
\hline non-circular/bottleneck & $127.1 /$ NA & 586.4 / NA \\
\hline non-circular/inverse & 169.5 / NA & 2226.8 / NA \\
\hline GMM/GMR & 127.8 / 75.9 \\
\hline Mean-Path & 99.6 / 75.3 \\
\hline
\end{tabular}

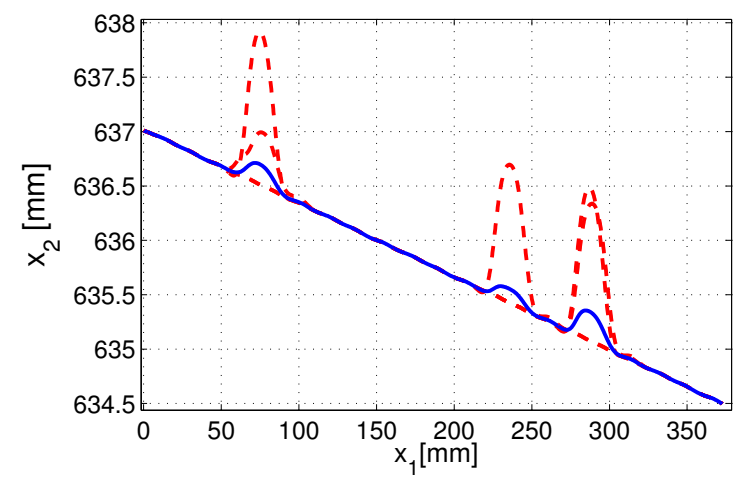

(a)

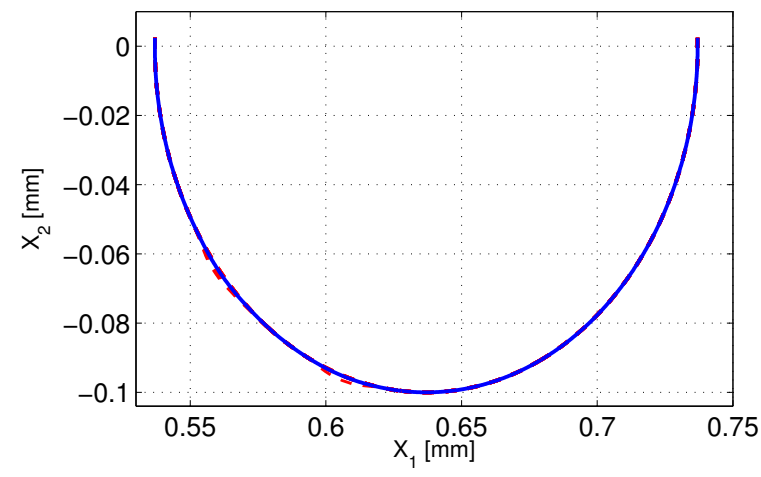

(b)

Fig. 5. (a) Ten linear profiles with randomly added artificial deburring residuals (red dashed lines), according to the models in Figure 4, and the computed mean profile (blue line). (b) Ten circular profiles with randomly added artificial deburring residuals (red dashed lines), and computed mean profile (blue line).

\section{CONCLUSION}

Human demonstrations are often sub-optimal and noisy solutions to the problem of performing a task. For many tasks, such as for example deburring, if a nominal trajectory, i.e. a model of the work-piece, is not available, a learning phase aiming at estimating it, is required. For this reason, we proposed an iterative algorithm to estimate a nominal path characterizing a task, given a set of noisy human demonstrations.

The effectiveness of the proposed algorithm is also shown considering a deburring example. Furthermore, a comparison with NLPCA and GMM/GMR demonstrates that the accuracy of these methods depends on the shape of the path, whereas the Mean-Path algorithm always results in approximately the same error, and its accuracy is always higher than the one achieved through the previous algorithms.

\section{REFERENCES}

[1] H. Zhang, H. Chen, N. Xi, G. Zhang, and J. He, "On-line path generation for robotic deburring of cast aluminum wheels," in IEEE/RSJ International Conference on Intelligent Robots and Systems, 2006, pp. 2400-2405.

[2] K. Shimokura and S. Liu, "Programming deburring robots based on human demonstration with direct burr size measurement," in IEEE International Conference on Robotics and Automation, 1994, pp. 572577. 


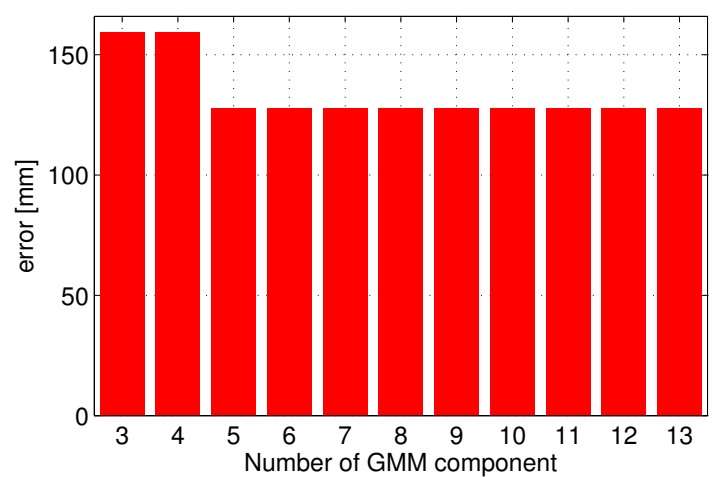

(a)

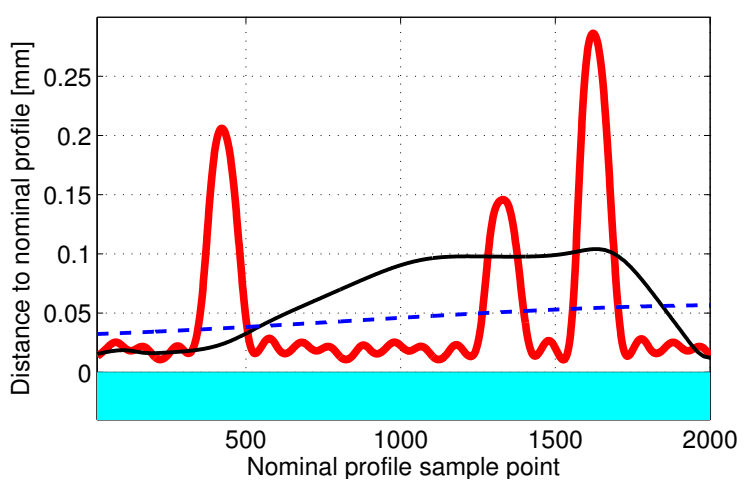

(b)

Fig. 6. The case of linear profiles: (a) sum of the distances, $\varepsilon$, between the linear nominal profile and the profile obtained using GMM/GMR; (b) the distances between the linear nominal profile and the paths computed using the Mean-Path algorithm (red thick line), GMM/GMR with 10 components (black line) and NLPCA (blue dashed line). The shaded green area represents the work-piece.

[3] M. M. Daniali and G. Vossoughi, "Intelligent active vibration control of constrained manipulaors in robotic deburring," in International Conference on Industrial Mechatronics and Automation, 2009, pp. 7680.

[4] G. Ziliani, A. Visioli, and G. Legnani, "A mechatronic approach for robotic deburring," Mechatronics, vol. 17, no. 8, pp. 431-441, 2007.

[5] E. Aertbelien and H. Van Brussel, "An observation model and segmentation algorithm for skill acquisition of a deburring task," in IEEE/ASME International Conference on Advanced Intelligent Mechatronics, 1999, pp. 635-640.

[6] F.-Y. Hsu and L.-C. Fu, "Intelligent robot deburring using adaptive fuzzy hybrid position/force control," IEEE Transactions on Robotics and Automation, vol. 16, no. 4, pp. 325-335, 2000.

[7] C.-P. Kuan and K.-Y. Young, "Reinforcement learning and robust control for robot compliance tasks," Journal of Intelligent and Robotic Systems, vol. 23, no. 2-4, pp. 165-182, 1998.

[8] K.-T. Song and T.-S. Chu, "Reinforcement learning and its application to force control of an industrial robot," Control Engineering Practice, vol. 6, no. 1, pp. 37-44, 1998.

[9] S. Calinon, Robot Programming by Demonstration. EPFL Press, 2009.

[10] S. Calinon, F. Guenter, and A. Billard, "On learning, representing, and generalizing a task in a humanoid robot," IEEE Transactions on Systems, Man, and Cybernetics, Part B: Cybernetics, vol. 37, no. 2, pp. 286-298, 2007.

[11] S. Cho and S. Jo, "Incremental online learning of robot behaviors from selected multiple kinesthetic teaching trials," IEEE Transactions on Systems, Man, and Cybernetics, Part A: Systems, vol. 43, no. 3, pp. 730-740, 2013.

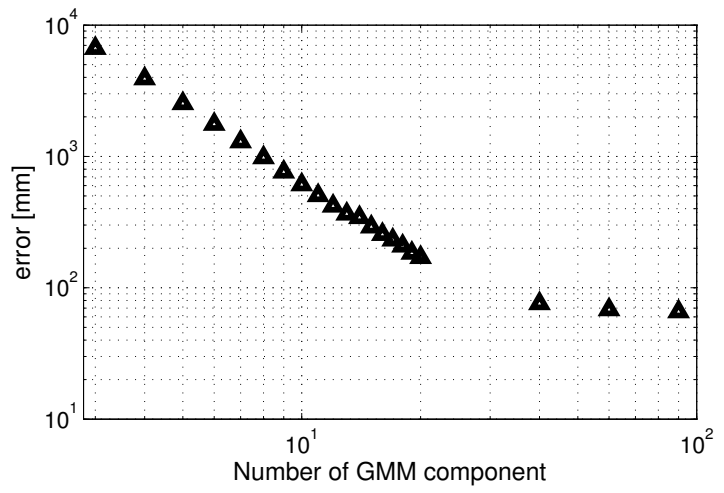

(a)

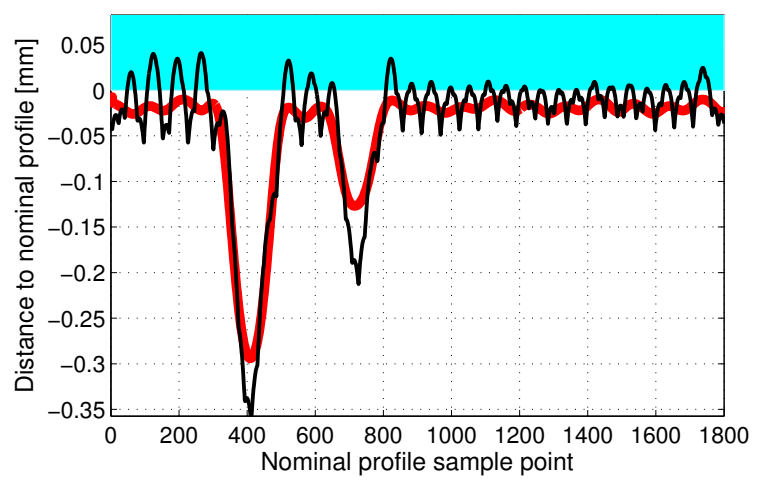

(b)

Fig. 7. The case of ten circular profiles: (a) sum of the distances, $\varepsilon$, between the circular nominal profile and the profile obtained using GMM/GMR; (b) the distances between the nominal profile and the paths computed using the Mean-Path algorithm (red thick line) and by GMM/GMR with 60 components (black line). The shaded green area represents the work-piece.

[12] M. Scholz, M. Fraunholz, and J. Selbig, "Nonlinear principal component analysis: neural network models and applications," in Principal manifolds for data visualization and dimension reduction. Springer, 2008, pp. 44-67.

[13] M. Scholz, "Validation of nonlinear PCA," Neural processing letters, vol. 36, pp. 21-30, 2012. 